\title{
WHAT A TANGLED WEB WE WEAVE: HERMUS AS THE NORTHERN EXTENSION OF THE PHOENIX STREAM
}

\author{
Carl J. Grillmair ${ }^{1}$ and Raymond G. Carlberg ${ }^{2}$ \\ ${ }^{1}$ Infrared Processing and Analysis Center, California Institute of Technology, Pasadena, CA 91125, USA; carl@ipac.caltech.edu \\ 2 Department of Astronomy and Astrophysics, University of Toronto, Toronto, ON M5S 3H4, Canada; carlberg@astro.utoronto.ca \\ Received 2016 February 15; accepted 2016 March 5; published 2016 March 23
}

\begin{abstract}
We investigate whether the recently discovered Phoenix stream may be part of a much longer stream that includes the previously discovered Hermus stream. Using a simple model of the Galaxy with a disk, bulge, and a spherical dark matter halo, we show that a nearly circular orbit, highly inclined with respect to the disk, can be found that fits the positions, orientations, and distances of both streams. While the two streams are somewhat misaligned in the sense that they do not occupy the same plane, nodal precession due to the Milky Way disk potential naturally brings the orbit into line with each stream in the course of half an orbit. We consequently consider a common origin for the two streams as plausible. Based on our best-fitting orbit, we make predictions for the positions, distances, radial velocities, and proper motions along each stream. If our hypothesis is borne out by measurements, then at $\approx 183^{\circ}\left(\approx 235^{\circ}\right.$ with respect to the Galactic center $)$ and $\approx 76 \mathrm{kpc}$ in length, Phoenix-Hermus would become the longest cold stream yet found. This would make it a particularly valuable new probe of the shape and mass of the Galactic halo out to $\approx 20 \mathrm{kpc}$.
\end{abstract}

Key words: Galaxy: halo - Galaxy: structure

\section{INTRODUCTION}

Recent years have seen the discovery of dozens of stellar debris streams in our Galaxy (see Grillmair \& Carlin 2016 and Smith 2016 for reviews). By virtue of their very low velocity dispersions, the cold stellar streams we believe to be the remnants of globular clusters are particularly well suited to the task of constraining the shape and size of the Galactic potential. On the other hand, such streams are far less populous than dwarf galaxy streams such as Orphan or Sagittarius and are consequently much harder to detect. Perhaps for this reason, we have yet to find a cold stream that even approaches the length of the Sagittarius stream.

Spanning $\approx 70^{\circ}$, GD-1 is the longest of the cold streams discovered to date (Grillmair \& Dionatos 2006; Carlberg \& Grillmair 2013), though at a distance of $\simeq 10 \mathrm{kpc}$ this corresponds to only $12 \mathrm{kpc}$ in length. Fitting orbits, Koposov et al. (2010) used GD-1 to put significant constraints on the circular velocity at the Sun's radius, though they were rather insensitive to halo flattening due to the proximity of the disk. Eyre \& Binney (2011) noted that orbit fitting is not generally appropriate for stellar streams in realistic potentials, as stars in streams do not follow a single orbit. Techniques have recently been developed to quickly generate mock tidal streams without a full $\mathrm{N}$-body treatment and to match these steams directly to observations (Küpper et al. 2012; Lane et al. 2012; Fardal et al. 2015). Using such techniques, Küpper et al. (2015) have once again demonstrated the potential of cold streams as true "high-precision scales" of the Galactic mass distribution.

Our expectation is that globular cluster streams should generally be very long. Since tidal stripping will have begun virtually as soon as globular clusters were born, then in the absence of major mergers (Wyse 2009), many streams will have had nearly the age of the universe to grow in length. Perturbations by dwarf galaxies, dark matter subhalos, or disk structures can generate significant gaps in streams (Carlberg 2009; Yoon et al. 2011), or even decollimate large parts of them to the point of undetectability. However, the existence of
GD-1 demonstrates that such events are not common enough to shorten or destroy all streams, at least in the inner halo.

From the work of Küpper et al. (2015) and others, we know that the constraints that can be put on the shape and size of the Galactic potential depend strongly on the length of a stream. We would clearly benefit from the discovery of cold streams that extend completely around the Galaxy, and at large distances from the Galactic center.

In this Letter, we test the hypothesis that the recently discovered Phoenix and Hermus streams may be part of the same structure. We use a simple model of the Galaxy to fit the streams in Section 2. Based on our best fit to the streams, we make predictions on observable quantities in Section 4. Concluding remarks are given in Section 5.

\section{ANALYSIS}

\subsection{Phoenix}

Balbinot et al. (2015, hereafter B16) recently discovered a cold stream in the Dark Energy Survey Year 1 data that they dubbed the Phoenix stream. With a width of only $54 \mathrm{pc}$, this $17.5 \mathrm{kpc}$ distant, $8^{\circ}$ long stream is very similar to the Pal 5 stream and presumably also originated in a globular cluster. From the color-magnitude distribution of its stars, B16 estimate an age of $11.5 \mathrm{Gyr}$ and a metallicity of $[\mathrm{Fe} / \mathrm{H}]$ $<-1.6$. Among the several overdensities along the stream, B16 find a pair of peaks somewhat out of alignment with the rest of the stream that they suggest may be the remnant of the progenitor. If true, then the positions of these peaks suggest that the stream is moving from south to north, in a prograde orbit around the Galaxy.

\subsection{Hermus}

Grillmair (2014, hereafter G14) discovered a pair of nearly parallel streams in the northern footprint of data release 10 of the Sloan Digital Sky Survey (SDSS; Ahn et al. 2014) that he dubbed Hermus and Hyllus. Both streams appear to be metal 


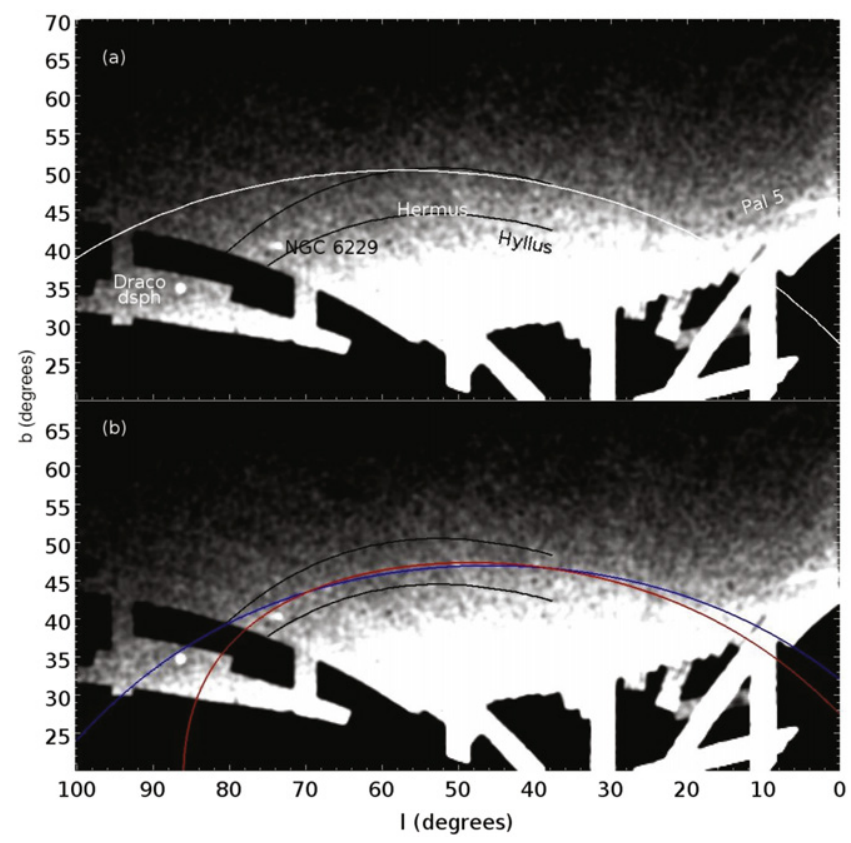

Figure 1. Panel (a): filtered surface density map of the western portion of the northern SDSS footprint, in Galactic coordinates. The stretch is linear, with lighter areas indicating higher surface densities. The map is the result of a filter based on the color-magnitude distribution of stars in the globular cluster M 53 and shifted to a distance of $20 \mathrm{kpc}$. The map has been smoothed with a Gaussian kernel of 0.6 . The black curves correspond to the Hermus trajectory of G14, offset $\pm 3^{\circ}$ in $b$. The white curve is the Galactocentric great circle fit to Phoenix by B16. Panel (b): the same map with best-fit orbits shown. The red curve shows an orbit fit to only the northern $30^{\circ}$ of Hermus, while the blue curve shows a simultaneous fit to both the Hermus and Phoenix streams.

poor, though the color-magnitude distributions are very noisy and $\mathrm{G} 14$ was unable to rule out $[\mathrm{Fe} / \mathrm{H}]$ as high as -1.2 for Hermus. G14 estimated Hermus to be about $20 \mathrm{kpc}$ distant, with the northernmost end of the stream perhaps as close as $15 \mathrm{kpc}$. While the stream appears to be some $50^{\circ}$ long (limited on both ends by limits of the SDSS survey footprint), G14 noted that the southern $20^{\circ}$ had a somewhat different character and curvature than the northern $30^{\circ}$, angling back toward the west and becoming somewhat stronger and broader at the southern end.

\section{PHOENIX-HERMUS?}

To search for possible Phoenix progenitors, B16 fit a great circle that contained both the Phoenix stream and the Galactic center. They found no globular clusters with similar distances along this great circle and concluded that the stream could not have originated from among the known clusters. In panel (a) of Figure 1, we show this great circle overplotted on the portion of the SDSS footprint containing Hermus. We see that the great circle passes less than $3^{\circ}$ from Hermus and that it is fairly well aligned with a significant portion of the stream.

For the purposes of demonstrating plausibility, we generate orbits using the Galactic model of Allen \& Santillan (1991). While assuming a spherical bulge and halo, this model also includes a disk and is therefore somewhat aspherical at low $|Z|$. As noted by Eyre \& Binney (2011), stellar streams are not expected to follow single orbits in realistic potentials. On the other hand, the deviations for cold, weakly stripped globular cluster streams in the nearly spherical potential of the inner halo (Küpper et al. 2015) are not expected to be large.
In panel (b) of Figure 1, we show a least-squares fit of an orbit to the R.A., decl., and distances of the northern $30^{\circ}$ of Hermus. For constraining the orbit, we do not use the southern $20^{\circ}$, which departs both from the great circle of B16 and from the curvature shown by the northern $30^{\circ}$. G14 noted the different character of the southern $20^{\circ}$ of Hermus, particularly the change in curvature of this portion of the stream. While not a definitive test, we note that an orbit fit to the entire $50^{\circ}$ of Hermus yields a reduced $\chi^{2}$ several times higher than a similar fit to just the northern $30^{\circ}$, with systematic departures in position and distance at the southern end. We therefore admit the possibility that the southern $20^{\circ}$ of Hermus is an unassociated structure at the same distance, and that G14 mistakenly assumed it to be part of the same structure. This would be in keeping with a common and perhaps natural tendency to ascribe the complexity of many small features to a comparatively simple, single stream.

As in G14, we assume uncertainties of $0^{\circ} .3$ in the 10 R.A., decl. positions along the stream, and $3 \mathrm{kpc}$ uncertainties in the distances at each point. This orbit fit is shown as the red curves in Figures 1 and 2. Nodal precession of the orbit due to the Galactic disk naturally brings the Hermus orbit into the plane occupied by Phoenix. The orbit fit misses Phoenix by several kiloparsecs, but this is entirely attributable to the distance gradient assumed for Hermus. Assuming a uniform distance of $20 \mathrm{kpc}$ for every point in Hermus, we find that a Hermus-only fit lies only $1-2 \mathrm{kpc}$ from the Phoenix stream. Reexamining G14's filtered surface density map, we find that his $15 \mathrm{kpc}$ estimate at the northern end of Hermus relies on some very faint structures that may or may not be part of the stream. A uniform $20 \mathrm{kpc}$ distance along the northern $30^{\circ}$ appears almost equally consistent with the data.

Encouraged by these apparent planar alignments, we now include 10 positions along the Phoenix stream in the orbit fit, measured from Figure 3 of B16. We use 10 positions to give equal weight to both Hermus and Phoenix. Just as for Hermus, we adopt 0.3 uncertainties for the positions along the stream, and $3 \mathrm{kpc}$ uncertainties in the distances at each point. The latter would combine to give the $0.9 \mathrm{kpc}$ uncertainty claimed by B16 for the entire stream. We also adopt the distance gradient found by B16, with Phoenix being $\approx 1 \mathrm{kpc}$ closer at its northern end than at the southern end.

This simultaneous fit to the Hermus and Phoenix streams is shown as the blue curves in panel (b) of Figure 1 and in Figure 2. We see that a single orbit is capable of closely fitting both streams. While the fit is nearly perfect for Phoenix (including both sky position and distance gradient), the trajectory in Figure 1 is slightly less curved than the Hermus stream itself. The maximum deviation between the model and the stream is $\approx 1.5$ at the extreme northern end. Given the simplicity of our Galactic model, we do not consider this very significant. It may be that a slightly prolate or substructured halo could easily accommodate the curvature of Hermus.

Similar attempts to simultaneously fit the Hyllus and Phoenix streams are much less interesting. Though Hyllus lies only $4^{\circ}$ east of Hermus and appears reasonably well aligned with B16's great circle, the minimum reduced $\chi^{2}$ is four times larger than for Hermus. Orbital precession is evidently insufficient to match the trajectories of both streams simultaneously, and the best-fitting orbits are clearly out-of-plane for one or the other, typically missing by several kiloparsecs. 


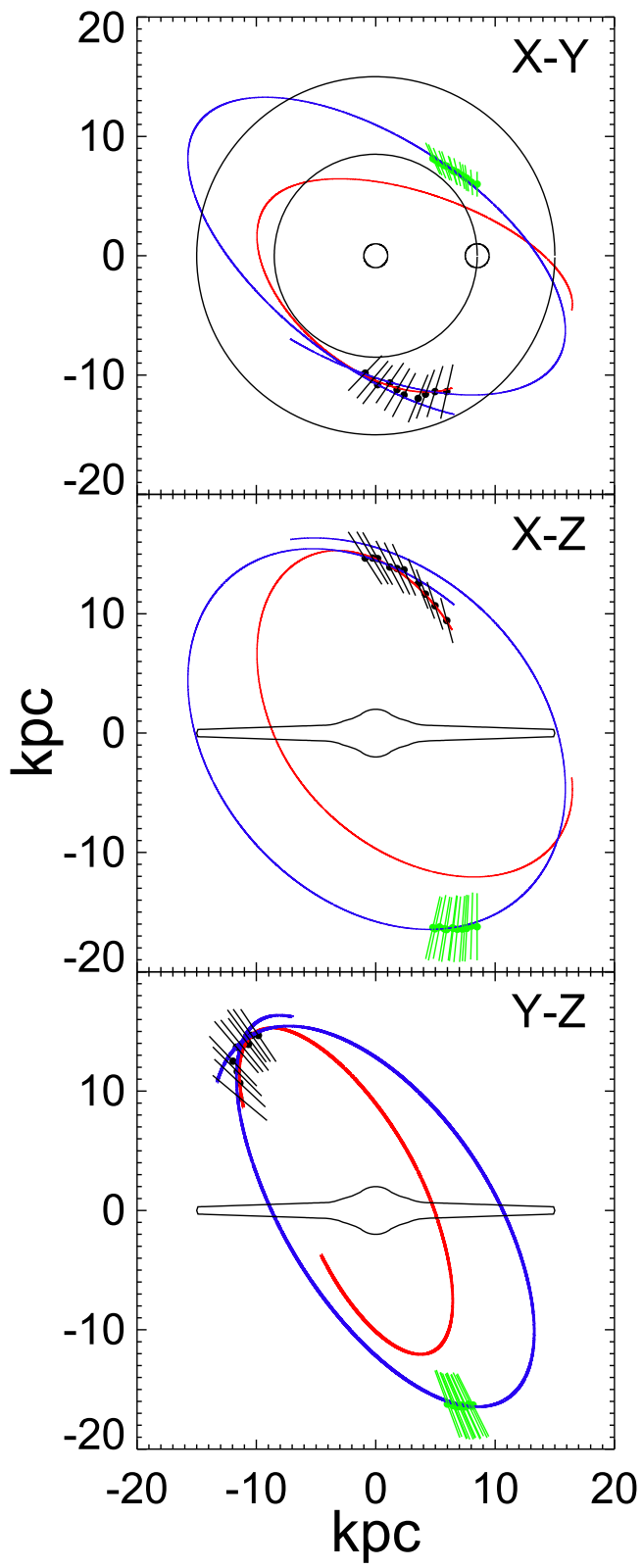

Figure 2. Projections of best-fit orbits in Galactic Cartesian coordinates. The solar circle and the position of the Sun are indicated. The path of the Hermus stream is shown by the black points, where the error bars correspond to $\pm 3 \mathrm{kpc}$. The green points and error bars indicate the path of the Phoenix stream. The red curve is an orbit fit solely to Hermus, while the blue curve shows a simultaneous fit to both Hermus and Phoenix. Note that orbital precession due to the disk is required to bring the orbit into alignment with the Phoenix stream.

The orbit that simultaneously fits Hermus and Phoenix is fairly circular, with apo- and perigalactica of $19.3_{-0.4}^{+1.7}$ and $17.6_{-0.8}^{+0.2} \mathrm{kpc}$ and an eccentricity of only $\approx 0.05$. This is somewhat surprising, given the roughly isotropic orbital distribution seen among globular clusters. Moreover, it argues that the progenitor of a putative Phoenix-Hermus stream must have been rather loosely bound to have been so significantly depleted in such a relatively benign orbit. The orbit is inclined $\approx 60^{\circ}$ to the Galactic plane, and the Phoenix stream is situated very nearly at the apogalactic point of the orbit. This would be qualitatively consistent with the apparent fading out of the Phoenix stream at its northern end, where the stars are picking up speed and the stream consequently becomes more tenuous.

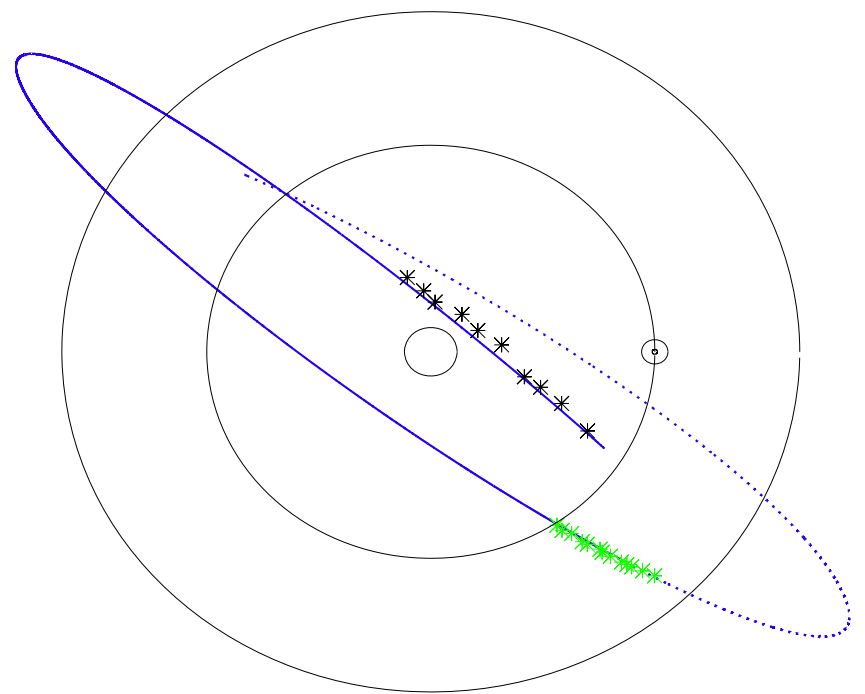

Figure 3. Projection of the best-fit orbit viewed nearly in the orbital plane. The leading (dotted) and trailing (solid) tails are at nearly the same radius, but are inclined by $\approx 9^{\circ}$ to one another due to nodal precession. Hermus is shown by black asterisks while Phoenix is shown in green. Scale and orientation are provided by the solar circle, with the Sun at $x=8.5 \mathrm{kpc}$.

B16 found two overdensities in Phoenix that are slightly out of alignment with the bulk of the stream and suggest that these overdensities could be the remnants of the progenitor. If true, then the arrangement of these overdensities implies that the stream must be moving from south to north and is in a prograde orbit around the Galaxy. The orbit that best fits both streams passes south from Hermus, through the disk on the far side of the Galactic center, within $15^{\circ}$ of the south celestial pole, and then north to Phoenix. If Phoenix and Hermus are related, then Hermus would constitute the trailing tail of the Phoenix stream. From the northern end of the leading (north) arm of the Phoenix stream to the trailing (northern) end of the Hermus stream, the best-fit orbit subtends $183^{\circ}$ on the sky and $76 \mathrm{kpc}$ through space. Viewed from the Galactic center, the orbit would subtend $235^{\circ}$, or two-thirds of a complete wrap around the Galaxy.

The leading arm passes north from Phoenix, through the anticenter portion of the disk, within $10^{\circ}$ of the north celestial pole, and then south to Hermus. However, nodal precession causes its path to deviate $\approx 9^{\circ}$ from the orbital plane of the stream. This is shown in Figure 3, where we show a nearly edge-on view of the orbit.

Owing to the near-circularity of the best-fit orbit, the leading arm ends up at nearly the same distance and nearly parallel to Hermus in the SDSS footprint. It lies $10^{\circ}$ west of Hermus on the northern end (right-hand side) of Figure 1 but converges with Hermus near Pal 5. We have carefully examined this region, and while there are some faint features that roughly line up with the predicted orbit of the leading arm, we find nothing we would have identified as a stream at the outset. This could be an indication that the leading and trailing arms are of different lengths, that the leading arm is weaker than the trailing arm, or that the leading arm has been dispersed by an encounter with one or more massive substructures. Of course, it could also be an indication that Hermus and Phoenix are not physically associated.

We have compared our best-fitting orbit with the positions of all known Galactic globular clusters. The closest matches are 


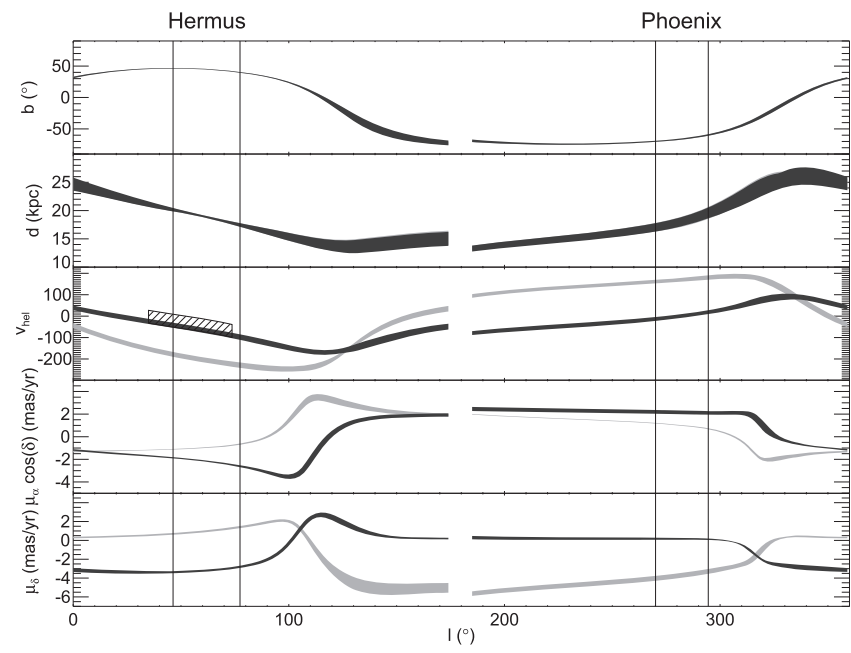

Figure 4. Predictions of our model orbit for various observables, as a function of Galactic longitude. The shaded regions encompass the $90 \%$ confidence interval in each case. The dark gray shaded regions correspond to a prograde orbit, while the lighter gray regions indicate expectation values for a retrograde orbit. The endpoints of the Hermus and Phoenix streams are shown by the vertical lines. The hashed box in the middle panel shows the $v_{G S R}$ limits used by C. Martin et al. (2016, in preparation) in kinematically detecting Hermus with blue horizontal branch stars. In the case of a prograde orbit, both streams would be moving toward lower longitudes, while in the retrograde case they would be moving to the right toward higher 1 .

with Pal 1, NGC 1261, and Pal 5. NGC 1261 and Pal 5 are clearly ruled out by B16 and Figure 1 of this work. Similarly, Pal 1 lies $\approx 2.3 \mathrm{kpc}$ laterally from the nearest branch of the orbit. Owing to Pal 1's proximity to the Sun, the best-fitting orbit passes no closer than $12^{\circ}$ from the cluster. We conclude that none of the known globular clusters is likely to be the progenitor of this stream.

\section{PREDICTED OBSERVABLES}

If Hermus and Phoenix are indeed part of the same stream, then our orbit model can be used to make approximate predictions for the radial and tangential velocities we would expect to measure. Figure 4 shows positions, distances, radial velocities, and proper motions as a function of Galactic longitude for both prograde and retrograde orbits. We show only the portion of the orbit connecting the streams on the far side of the Galactic center, which is the arm that, via nodal precession, correctly predicts the orientations of both Hermus and Phoenix.

Uncertainties were estimated using the marginal $\chi^{2}$ distributions for $v_{\text {hel }}, \mu_{\alpha} \cos (\theta)$, and $\mu_{\delta}$. Each parameter was offset to its $90 \%$ confidence limit and the other fit parameters were then varied to find a new $\chi^{2}$ minimum. The shaded regions in Figure 4 encompass the entire range of observable parameters resulting from this procedure.

Also shown in Figure 4 are Galactic standard of rest velocity cuts used by C. Martin et al. (2016, in preparation) to kinematically identify Hermus using blue horizontal branch stars. The velocities clearly favor a prograde orbit for Hermus and, if the Phoenix and Hermus streams are part of the same stream, are completely consistent with our predictions for a prograde orbit.

\section{CONCLUSIONS}

Having found that the Hermus and Phoenix streams are nearly coplanar, we have investigated whether a single orbit could accommodate both streams. Using a Galactic model with a disk, bulge, and spherical halo, we find that we can indeed match the trajectories of both streams with a single orbit. Moreover, this match is partly facilitated by orbital precession, which naturally brings the orbital plane into alignment with each stream within half an orbit around the Galaxy.

B16 identify a possible progenitor within the Phoenix stream. The misalignment of this feature with the stream itself suggests that Phoenix is on a prograde orbit around the Galaxy. If Phoenix and Hermus are part of the same stream, then Hermus must be a remote part of the trailing arm.

While not proven, we consider the hypothesis that Hermus and Phoenix are part of the same stream as entirely plausible. Confirmation will require spectroscopy of stars in both Hermus and Phoenix. The kinematic discovery of Hermus (C. Martin et al. 2016, in preparation) already supports a prograde orbit for the stream and is entirely consistent with our predictions based on a single-stream orbit.

If radial velocity measurements and/or proper motions confirm a physical association between the Hermus and Phoenix streams, then as the longest cold stream yet discovered, Phoenix-Hermus would provide us with a remarkable new probe of the Galactic potential.

We thank an anonymous referee for several useful suggestions that improved both the clarity and thoroughness of the manuscript.

\section{REFERENCES}

Ahn, C. P., Alexandroff, R., Allende Prieto, C., et al. 2014, ApJS, 211, 17 Allen, C., \& Santillan, A. 1991, RMxAA, 22, 255

Balbinot, E., Yanny, B., Li, T. S., et al. 2016, ApJ, 820, 58 (B16)

Carlberg, R. G. 2009, ApJ, 705, 223

Carlberg, R. G., \& Grillmair, C. J. 2013, ApJ, 768, 171

Eyre, A., \& Binney, J. 2011, MNRAS, 413, 1852

Fardal, M. A., Huang, S., \& Weinberg, M. D. 2015, MNRAS, 452, 301

Grillmair, C. J. 2014, ApJL, 790, L10 (G14)

Grillmair, C. J., \& Carlin, J. L. 2016, in Tidal Streams in the Local Group and Beyond, ed. H. J. Newberg, \& J. L. Carlin (Berlin: Springer)

Grillmair, C. J., \& Dionatos, O. 2006, ApJ, 643, 17

Koposov, S., Rix, H.-W., \& Hogg, D. W. 2010, ApJ, 712, 260

Küpper, A. H. W., Balbinot, E., Bonaca, A., et al. 2015, ApJ, 803, 80

Küpper, A. H. W., Lane, R. R., \& Heggie, D. C. 2012, MNRAS, 420, 2700

Lane, R. R., Küpper, A. H. W., \& Heggie, D. C. 2012, MNRAS, 423, 2845

Smith, M. C. 2016, in Tidal Streams in the Local Group and Beyond, ed. H. J. Newberg, \& J. L. Carlin (Berlin: Springer)

Wyse, R. F. G. 2009, in Proc. IAU Symp. 258, The Ages of Stars, ed

E. E. Mamajek, D. R. Soderblom, \& R. F. G. Wyse (Cambridge: Cambridge Univ. Press), 11

Yoon, J. H., Johnston, K. V., \& Hogg, D. W. 2011, ApJ, 731, 58 